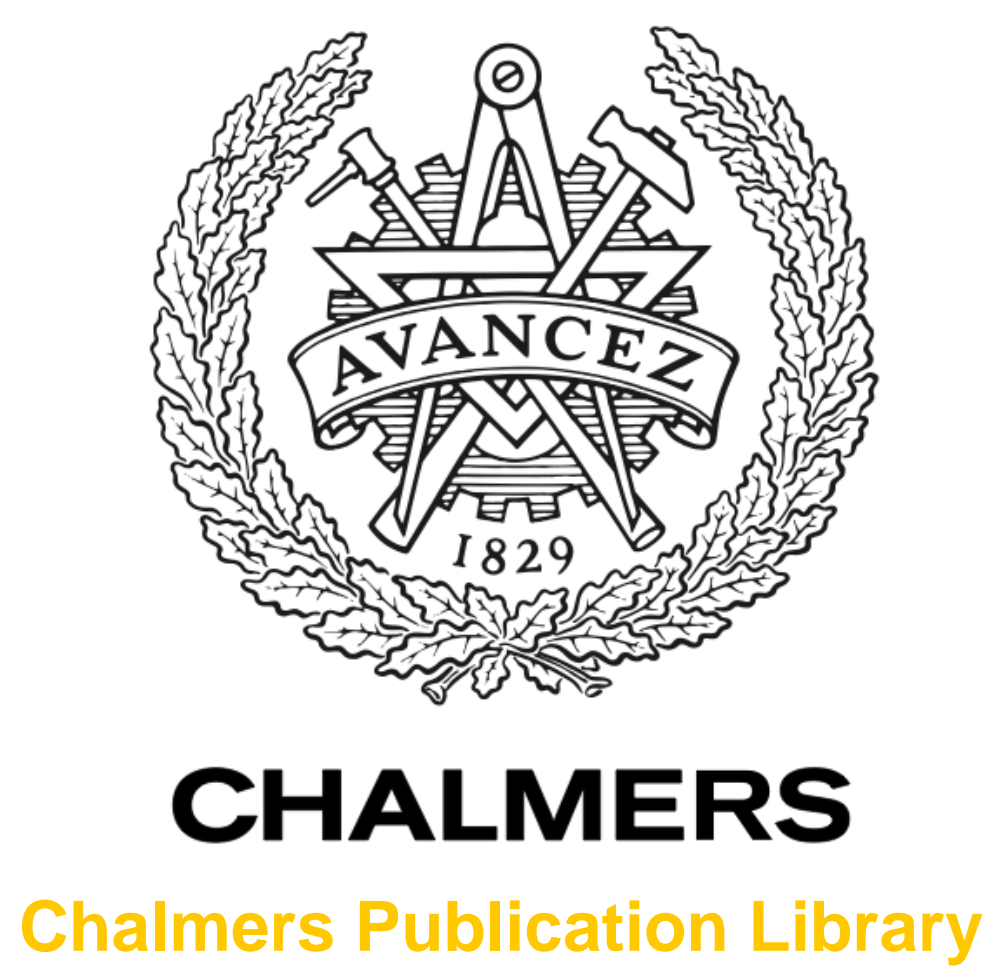

\title{
Investigation of brain tissue segmentation error and its effect on EEG source localization
}

This document has been downloaded from Chalmers Publication Library (CPL). It is the author's version of a work that was accepted for publication in:

\section{4th Annual International Conference of the IEEE Engineering in Medicine and Biology} Society, EMBS 2012, San Diego, CA, 28 August - 1 September 2012 (ISSN: 1557-170X)

Citation for the published paper:

Shirvany, Y. ; Porras Péres, A. ; Kowkabzadeh, K. (2012) "Investigation of brain tissue segmentation error and its effect on EEG source localization". 34th Annual International Conference of the IEEE Engineering in Medicine and Biology Society, EMBS 2012, San Diego, CA, 28 August - 1 September 2012 pp. 1522-1525.

http://dx.doi.org/10.1109/EMBC.2012.6346231

Downloaded from: http://publications.lib.chalmers.se/publication/169568

Notice: Changes introduced as a result of publishing processes such as copy-editing and formatting may not be reflected in this document. For a definitive version of this work, please refer to the published source. Please note that access to the published version might require a subscription. 


\title{
Investigation of Brain Tissue Segmentation Error and its Effect on EEG Source Localization
}

\author{
Yazdan Shirvany*, Student Member, IEEE, Antonio R. Porras, Koushyar Kowkabzadeh, Qaiser Mahmood, \\ Hoi-Shun Lui, Member, IEEE, and Mikael Persson, Member, IEEE
}

\begin{abstract}
Surgical therapy has become an important therapeutic alternative for patients with medically intractable epilepsy. Correct and anatomically precise localization of the epileptic focus, preferably with non-invasive methods, is the main goal of the pre-surgical epilepsy diagnosis to decide if resection of brain tissue is possible. For evaluating the performance of the source localization algorithms in an actual clinical situation, realistic patient-specific human head models that incorporate the heterogeneity nature of brain tissues is required. In this paper, performance of two of the most widely used software packages for brain segmentation, namely FSL and FreeSurfer has been analyzed. Then a segmented head model from a package with better performance is used to investigate the effects of brain tissue segmentation in EEG source localization.
\end{abstract}

\section{INTRODUCTION}

Studies of the human brain have been of significant interest for medical doctors and neurosurgeons throughout the years. Functional magnetic resonance imaging (fMRI), electroencephalography (EEG) and integrated method, EEG/fMRI, have been the main tools for monitoring neural activities in many clinical studies. Recent development in computer hardware and computational physics provide an excellent platform for numerical modeling of these diagnostic tools, which allow us to develop semi-automated or even automated solutions for analyzing functional data and for diagnostic purposes, e.g., localizing epileptic brain activity.

In the past, due to the limitation of computer resources and numerical modelling techniques, only simplified spherical head models are used for brain activity source localization problems [1], [2]. As discussed in [3], for EEG source localization the CSF layer plays an important role in modifying the scalp potentials and it also affects the inverse source localization results. Moreover, Wolters et al. in [4] showed that anisotropic (different conductivity values in different space directions) skull and white matter conductivities affect the current flow distribution inside the head volume and the forward potential computation, respectively. Thus, if one would need to evaluate the performance of the source localization algorithms in an actual clinical situation, realistic patient-specific human head model that incorporates the heterogeneity nature of brain tissues and conductivities is required.

To build these realistic patient-specific head models, it is necessary to obtain all information about the tissues and anatomical structures between the signal source(s) and receiving electrodes. One way to obtain such information is to perform accurate segmentation based on anatomical brain MR images. After the anatomical information is extracted from the clinical MR images, we need to incorporate it to

\footnotetext{
*Authors are with the Department of Signals and Systems, Chalmers University of Technology and MedTechWest Center, Göteborg, Sweden. yazdan.shirvany at chalmers.se
}

build the model. With the advancement of computational physics together with computational resources, it is possible to spatially discretize the anatomical structure of the human brain and to simulate the entire brain activity source localization problem using finite element method (FEM) [5]. Using FEM, the heterogeneity nature of human brain is taken into account and realistic patient-specific head models are thus developed.

Out of these two steps for generating realistic head models, accurate brain segmentation is more important as the accuracies of the segmentation result would directly affect the accuracies for both forward and inverse problems for brain activity source localization. In the last two decades, many research groups have designed software packages for brain MRI data sequence analysis, reconstruction of the brain's cortical surface from anatomical MR data and registration of functional MR data on the reconstructed cortical surface. Among them, the most widely used are FMRIB Software Library (FSL) [6] and FreeSurfer [7].

The objective of this work is to investigate how the accuracies of the segmentation results could affect the accuracies in EEG source localization. To the best of our knowledge, such kind of studies has not been well investigated. As a first step, we consider simulated brain phantoms from BrainWeb [8] with "ground truth" (GT) in this study. Such numerical phantoms are available in the internet and they serve as a platform for evaluation of segmentation performance in the image analysis society. In this work, the BrainWeb phantoms are segmented using FSL and FreeSurfer and the corresponding segmentation results are evaluated. Then, FE models of the GT and segmented head models are built. The GT FE head model is used for generating EEG signals by putting an artificial source inside the model to mimic the clinical data from patients. The segmented head models are then used to perform the brain activity source localization. Here, we would like to investigate the performance of the source localization under imperfect tissue segmentation.

The paper is outlined as follows. Details about the evaluation tools of the segmentation results and the EEG source localization will be given in the next section, followed by some numerical results and discussions. Conclusions and future work will be reached towards the end of the paper.

\section{METHOD}

\section{A. Brain Tissue Segmentation}

Brain tissue segmentation of BrainWeb phantom is first performed independently using these two software packages. Comparison between the two sets of segmentation results is performed in a voxel-by-voxel basis by counting the number of true positives, false positives, true negatives and false negatives voxels for each tissue type. Having these measures as a starting point, the specificity, sensitivity and 


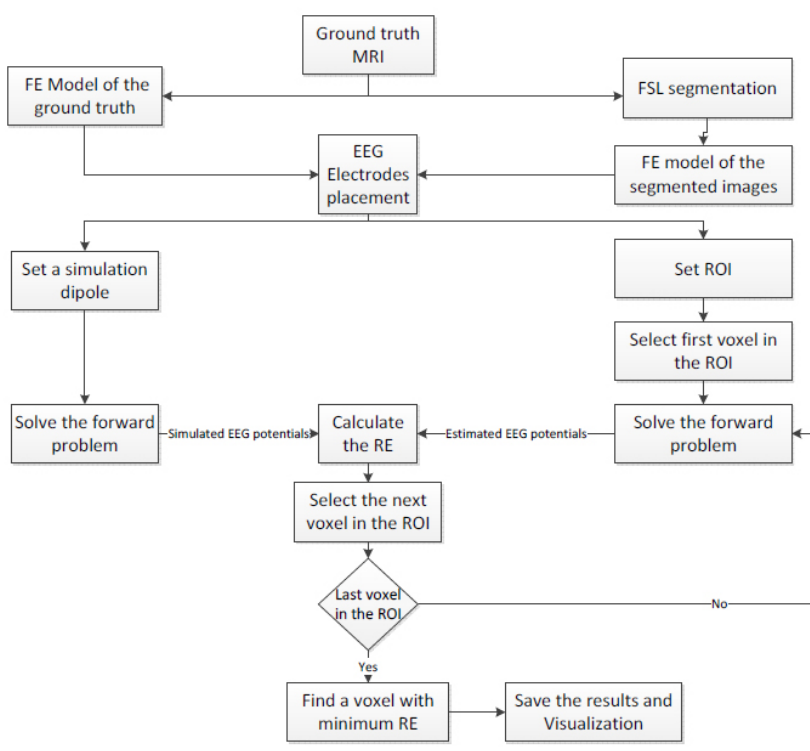

Fig. 1: Flowchart of the EEG source localization scenario. Note: ROI stands for region of interest.

mis-classification ratio (MCR) are computed [9], [10], [11], [12], which are defined as

$$
\begin{gathered}
\text { Specificity }=\frac{\text { True_Negatives }}{\text { True_Negatives }+ \text { False_Positives }} \times 100 \% \\
\text { Sensitivity }=\frac{\text { True_Positives }}{\text { True_Positives }+ \text { False_Negatives }} \times 100 \% \\
M C R=\left(1-\frac{\text { volume }(A \cap B)}{\text { volume }(B)}\right) \times 100 \%
\end{gathered}
$$

where $\mathrm{A}$ is a segmented tissue and $\mathrm{B}$ is the GT tissue corresponding to A. Specificity measures the probability of correct classification of all voxels which are not part of the tissue. Sensitivity, on the other hand, measures the probability of correct classification of all voxels which belong to the tissue. MCR gives an idea about the percentage of voxels that has been incorrectly classified. The performance of the segmentation of these three software packages are evaluated and compared using these three parameters.

\section{B. EEG Source Localization}

Epilepsy is one of the most common neurologic diseases in the world and many patients with epilepsy never receive the treatment which make them seizure free. Surgical therapy has become an important therapeutic alternative for patients with medically intractable epilepsy. Source localization of epileptic activity is a tool to delineate cortical areas/volumes with abnormal neuronal activity of cells and networks [13]. However, correct and anatomically precise localization of the epileptic focus is mandatory to decide if resection of brain tissue is possible.

Source localization accuracy is affected by different factors including, head-modeling error, EEG signal noise and electrode displacements as well as the computational error. Among those the head model errors affect the scalp potentials and also influence the forward and inverse source localization results significantly [3], [4]. Roman et al. [3] showed that the model with five tissues, i.e., grey matter (GM), white matter (WM), cerebrospinal fluid (CSF), skull and skin, has

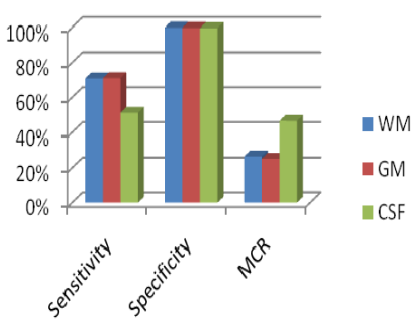

(a)
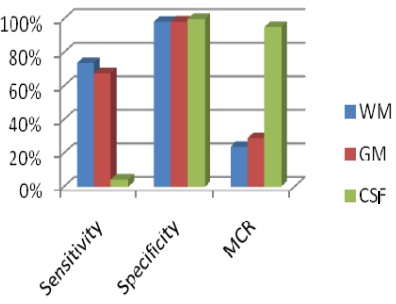

(b)
Fig. 2: Summary of the segmentation results for (a) FSL, (b) Freesurfer.

a good approximation of the head model for EEG source localization. Moreover, the number of tissues using in the head model and their accuracies are heavily depend on the brain tissue segmentation accuracy. To understand the effects of brain image segmentation on the EEG source localization, we set up a simulation scenario. This scenario is implemented in two steps and is shown in Fig. 1. Step 1) First, a FE head model was constructed from the GT MR slices and 61 scalp electrodes was placed on the head model surface based on 10/10 system [14]. Then, a simulation dipole source was placed in the somatosensory cortex of the GT FE head model and the synthetic EEG potentials were calculated at the electrode positions by solving the forward problem. Step 2) The inverse problem were solved for a fivetissue FE head model constructed from the segmented MR slices, i.e., GM, WM, CSF, skull and skin. In the inverse problem we do an exhaustive search pattern, i.e., inversion was performed for each possible source location in the motor and sensory cortex area inside a region of interest (ROI) and the site producing the smallest residual norm was selected as the best possible source location.

\section{A. Brain Image Segmentation}

The segmentation is performed over $18 \mathrm{~T} 1$-weighted MR phantoms obtained from BrainWeb with $1 \times 1 \times 1 \mathrm{~mm}$ resolution. These phantoms correspond to images with 6 different levels of noise $(0 \%, 1 \%, 3 \%, 5 \%, 7 \%$ and $9 \%)$ and 3 levels of radio-frequency in-homogeneities $(0 \%, 20 \%$ and $40 \%)$. Next, sensitivity, specificity and MCR were calculated to compare the results. In this sense, for each parameters (sensitivity, specificity and MCR), we obtained 18 different values for each tissue type from each software. We then take an average of the data to get an overall picture about the performance.

Summary of the segmentation results for FSL are shown in Fig. 2a. In this case, specificity is close to $100 \%$ for the three tissues, which means that FSL is good at classifying non-tissue elements. Moreover, for sensitivity, it is lower for all tissues, especially for CSF with the lowest value of $51 \%$. Since sensitivity is the ability to detect all voxels that are part of the tissue type, it means that FSL is able to detect $71 \%$ of voxels for GM and WM, and 51\% for CSF. Finally, if we look at MCR, we can see that for GM and WM, the MCR is about $25 \%$, but for CSF it is close to $46 \%$. Fig. 3, shows the difference between the segmented result and the GT for each tissue for the case of $1 \%$ RF noise and 20\% RF in-homogeneities.

There are some differences between output of FreeSurfer 


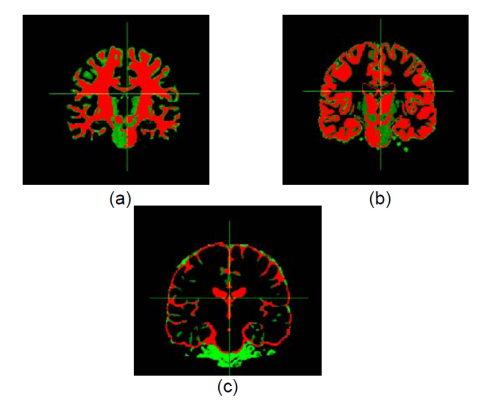

Fig. 3: Comparison between segmented tissues in FSL (red) and ground truth (green) for the $1 \%$ noise and $20 \% \mathrm{RF}$ in-homogeneities phantom. (a) WM, (b) GM and (c) CSF.

and FSL. Instead of having separate images for different tissue types, FreeSurfer creates a single image with all information with different intensities. For comparison purposes, it is important to extract the three tissue types (WM, GM and CSF) into three separate images. Second, the segmentation output from FreeSurfer results in 47 different tissues. These structures are classified into three tissue types by a clinical expert. Summary of the segmentation results for FreeSurfer are shown in Fig. 2b. If we consider the specificity for FreeSurfer, we can see that specificity is high and close to $100 \%$ for all the three tissues. However, sensitivity is lower for WM (73\%), GM (67\%), and much lower for CSF (4\%). This indicates that FreeSurfer is not able to segment CSF correctly. If we consider MCR, we can see that it is similar to those obtained in FSL for GM and WM, but it is much worse for CSF. Fig. 4, provides a visual view between the GT and the segmented output from Freesufer for the case with $1 \%$ noise and $20 \%$ RF in-homogeneities. It can easily be seen that the classification for CSF is wrong and part of the $\mathrm{WM}$ and GM is not segmented properly. The above findings show that FSL gives more reliable results for brain tissue segmentation, especially for CSF. As CSF has the important role in EEG source localization [3] FSL is chosen as the segmentation tool for EEG source localization in this work.

\section{B. EEG source localization}

We selected a T1-weighted BrainWeb MR phantom with $1 \times 1 \times 1 \mathrm{~mm}$ resolution, $3 \%$ noise and $20 \% \mathrm{RF}$ inhomogeneities. We tried to select a MR data set which had a level of noise comparable with a real noise-contaminated MR data. Fig. 5a, shows the cross section of the GT FE head model. This model has 8 tissues, i.e., GM, WM, CSF, fat, muscle, conductice tissue (CT), skull and skin. The following conductivities are assigned to the GT FE compartments based on their segmentation labels and the isotropic reference model [15]: $\mathrm{GM}=0.33 \mathrm{~S} / \mathrm{m}, \mathrm{WM}=0.142 \mathrm{~S} / \mathrm{m}, \mathrm{CSF}=1.538$

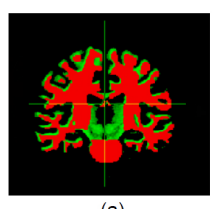

(a)

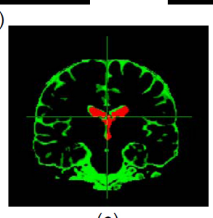

Fig. 4: Comparison between segmented tissues in Freesurfer (red) and ground truth (green) for the $1 \%$ noise and $20 \% \mathrm{RF}$ in-homogeneities phantom. (a) WM, (b) GM and (c) CSF.

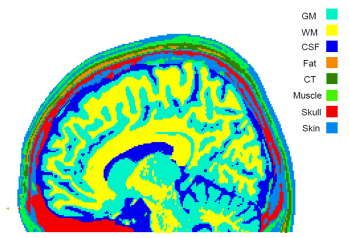

(a)

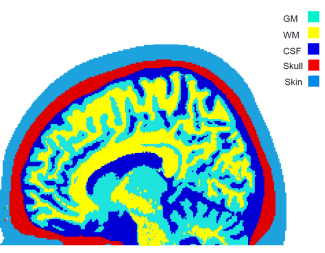

(b)
Fig. 5: The FE head model generated from a) ground truth, b) FSL segmented data. Note: CT stands for conductive tissue.

$\mathrm{S} / \mathrm{m}$, fat $=0.02081 \mathrm{~S} / \mathrm{m}$, muscle $=0.26671 \mathrm{~S} / \mathrm{m}, \mathrm{CT}=0.1628$ $\mathrm{S} / \mathrm{m}$, skin $=0.43 \mathrm{~S} / \mathrm{m}$ and skull $=0.0042 \mathrm{~S} / \mathrm{m}$ (skull to skin conductivity ratio of approximately $1: 100$ ).

The segmentation of the five tissues is done by FSL in two steps. In a first step, mask of skin, skull and brain is generated by using a preset intensity threshold value (ITV) in the BET module. In a second step, automated segmentation of three tissues i.e., GM, WM and CSF, of the brain is performed by applying the FAST module [6]. For the BET step different ITVs, i.e., 0.3, 0.4 and 0.5 , are tried but here we just present the results obtained from 0.3 which has the minimum source localization error. Fig. 5b shows a cross section of the fivetissue FSL FE head model for 0.3 threshold value. The assigned conductivities to FSL model are same as GT model. Comparison between GT and FSL FE head model in Fig. $5 \mathrm{a}$ and $5 \mathrm{~b}$ shows that the amount of CSF in FSL model is much more than the GT model. Moreover, fat, muscle and conductive tissue (CT) are mainly classified as skin in FSL model. Table I shows the tissue overlapping percentage [16] between the segmented results and the GT for each tissue. The ideal value in this table is $100 \%$ which means that a selected tissue is $100 \%$ same as the corresponding tissue in the GT MR image. As we can see in Table I the segmentation results for 0.4 and 0.5 are almost the same and consequently the EEG source localization result was the same as well.

Fig. 6, shows the results of the exhaustive search for the head model with intensity threshold value 0.3 . The results was plotted on the GT MR images. The yellow patch shows the position of the simulation source and the red patch indicates the position of a estimated source. As we can see in Fig. 6, the $\mathrm{x}$ - and $\mathrm{y}$-coordinates of the estimated source are correct and they correspond to the simulation source, while the $\mathrm{z}$-coordinate is deeper. The source localization error, distance between the simulation and estimated source, was $1.2 \mathrm{~cm}$. The results visualization was done in Slicer [17]. Fig. 7, shows the EEG potential generated by the simulation and estimated source at each individual sensor. The relative error between these two EEG potentials was 0.14.

Notice that we have used an exhaustive search pattern to localize the sources. This means that all the possible GM cells were searched in the $1 \mathrm{~mm}$ cubic volume of the motor and sensory cortex. The cell producing the least error was selected as the possible source location. This provides the best behavior of a given model in the inverse source TABLE I: Tissue overlapping percentage for three different intensity threshold values (ITV)

\begin{tabular}{|c||c||c||c|}
\hline & ITV $=0.3$ & ITV $=0.4$ & ITV $=0.5$ \\
\hline GM & $95.83 \%$ & $95.85 \%$ & $95.85 \%$ \\
\hline WM & $91.22 \%$ & $90.08 \%$ & $90.07 \%$ \\
\hline CSF & $76.13 \%$ & $73.20 \%$ & $73.18 \%$ \\
\hline
\end{tabular}




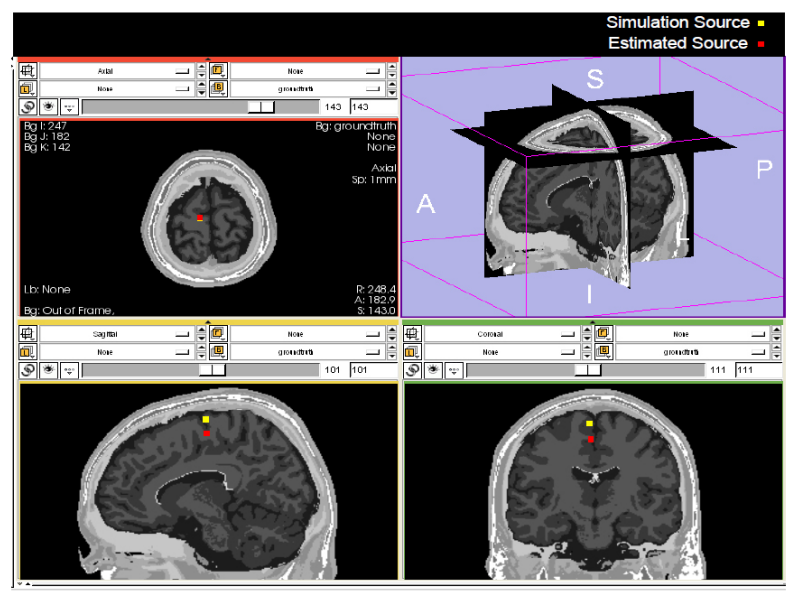

Fig. 6: The results of the exhaustive search plotted on the GT MR images. The yellow patch is the simulation source and the red patch is the estimated source. Note: The MRI space in Slicer is flipped compare to the anatomical space.

localizations and that mean this is the best result one could expect from a given model.

\section{CONCLUSION}

The accuracies of the brain tissue segmentation using FSL and FreeSurfer are evaluated and the impacts of how the segmentation accuracies could affect the performance of EEG source localization are investigated using simulated brain phantoms from BrainWeb. Comparing with the "ground truth", the results show that the segmentation results obtained from FSL give better accuracies than those from FreeSurfer.

A realistic head model is developed based on the segmentation results obtained from FSL and EEG source localization is performed using the FSL-segmented head model. The results show that the $\mathrm{x}$ - and $\mathrm{y}$-coordinates of the estimated source point is well located but not the $\mathrm{z}$-coordinate. This is probably due to the relatively large segmentation error in the CSF. Moreover, the brain extraction step can affect the CSF miss-classification significantly while the large amount of the CSF is placed in between the brain and skull compartments. During this pre-processing step, part of the CSF may be regarded as skull and skin mask. To handle this, we need to develop or seek alternative solutions for brain extraction and brain tissue segmentation that could result in a more accurate CSF classification. Furthermore, this work is performed using simulated BrainWeb phantoms. Future work should also consider using clinical MR images from patients.

It will be interested to compare the source localization performance using FE head models developed using "ground truth" segmentation result done by clinical experts verse segmentation tools such as those considered in this paper and also integrating different imaging modalities (e.g., T1,T2/Proton Density (PD) and Diffusion Tensor (DT)-MRI) to give a more accurate description about the anatomical structure of the head and conductivity of different tissues.

\section{ACKNOWLEDGMENT}

The authors would like to thank Assoc. Prof. Fredrik Edelvik, and Dr. Stefan Jakobsson from the FraunhoferChalmers Research Centre, Göteborg, Sweden for their contribution in the forward problem. The travel fund for this paper was granted by the Royal Society of Arts and Sciences

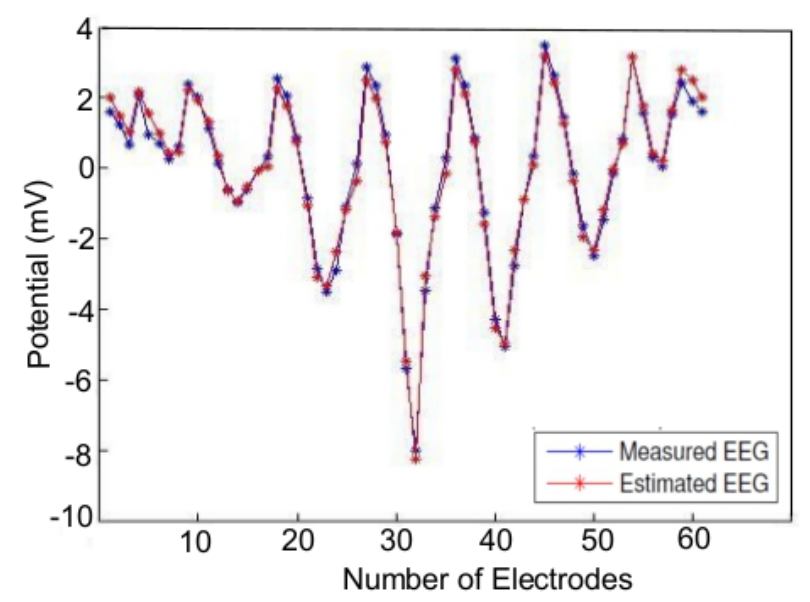

Fig. 7: Electrodes Potential for head model with intensity threshold value 0.3 , relative error between simulated and estimated EEG is 0.14 of Göteborg, Sweden.

\section{REFERENCES}

[1] E. Frank, "Electric potential produced by two point current sources in a homogeneous conducting sphere," Journal of Applied Physics, vol. 23, no. 11, pp. 1225-1229, 1952.

[2] Y. Salu, L. Cohen, D. Rose, S. Sxato, C. Kufta, and M. Hallett, "An improved method for localizing electric brain dipoles," Biomedical Engineering, IEEE Transactions on, vol. 37, no. 7, pp. $699-705$, 1990.

[3] C. Ramon, P. Schimpf, J. Haueisen, "Influence of head models on EEG simulations and inverse source localizations," BioMedical Engineering OnLine 2006

[4] C.H. Wolters, A. Anwander, X. Tricoche, D. Weinstei, M.A. Koch and R.S. MacLeod, "Influence of tissue conductivity anisotropy on EEG/MEG field and return current computation in a realistic head model: A simulation and visualization study using high-resolution finite element modeling," Journal of NeuroImage, Vol. 30, No. 3, pp. 813-826, 2005.

[5] M. Rullmann, A. Anwander, M. Dannhauer, S.K. Warfield, F. H. Duffy, C. H. Wolters, "EEG source analysis of epileptiform activity using a $1 \mathrm{~mm}$ anisotropic hexahedra finite element head model," NeuroImage, Vol. 44, pp. 399-410, 2009.

[6] S. M. Smith et al., "Advances in functional and structural MR image analysis and implementation as FSL," NeuroImage, vol. 23, pp. S208 - S219, 2004.

[7] FreeSurfer. [Online], http://surfer.nmr.mgh.harvard.edu/.

[8] C.A. Cocosco, V. Kollokian, R.K.-S. Kwan and A.C. Evans: "BrainWeb: Online Interface to a 3D MRI Simulated Brain Database" NeuroImage, vol.5, no.4, part 2/4, S425, 1997 - Proceedings of 3-rd International Conference on Functional Mapping of the Human Brain, Copenhagen, May 1997.

[9] R. Baeza-Yates, B. Ribeiro-Neto, Modern information retrieval. s.1. : ACM Press, 1999. 020139829

[10] D. L. Olson, D. Delen, Advanced Data Mining Techniques. s.l. Springer, 2008.

[11] T. Song, M. M. Jamshidi, R. R. Lee, M. Huang, "A Modified Probabilistic Neural Network for Partial Volume Segmentation in Brain MR Image," IEEE Trans. Neural Networks, Vol. 18, No. 5, pp. 1424-1432, 2007.

[12] O. Tsang, A. Gholipour, N. Kehtarnavaz, K. Gopinath, R. Briggs, I. Panahi, "Comparison of tissue Segmentation Algorithms in Neuroimage Analysis Software Tools," Proc. of 30th annual conference of EMBS, British Columbia , Canada, Aug. 20-24, 2008.

[13] D. L. Kraeme, M. E. Brandling-Bennett, and D. G. Vossler. Epilepsy surgery. Medscape reference, Drug, Diseases and Procedures, 2011.

[14] V. Jurcak, D. Tsuzuki, and I. Dan, "10/20, 10/10, and 10/5 systems revisited: Their validity as relative head-surface-based positioning systems," Neurolmage, vol. 34, no. 4, pp. 1600 - 1611, 2007.

[15] S. Gabriel, R. Lau, and C. Gabriel, "The dielectric properties of biological tissues: III parametric models for the dielectric spectrum of tissues," Phys Med Biol, vol. 41, no. 11, pp. 2271 - 2293, 1996.

[16] Dice, Lee R. (1945). "Measures of the Amount of Ecologic Association Between Species". Ecology 26 (3): 297302

[17] S. Pieper, M. Halle, and R. Kikinis, "3D SLICER." Proceedings of the 1st IEEE International Symposium on Biomedical Imaging: From Nano to Macro, 2004, pp. 632-635. 